\section{Navigate the Omicron challenge}

As the Omicron challenge looms over the public sector and the risk of up to $25 \%$ staff absence remains prevalent, Dentally wants to ensure that you, your practice team, and your patients have the tools in place to cope with any unexpected periods of isolation. From late cancellations to working remotely, Dentally can help minimise the impact the surge in infection rates has on daily practice life and help you navigate the challenges of the current climate.

The government has asked the public sector to add contingency plans against a worst-case scenario of $25 \%$ staff absence, as part of the efforts to minimise disruption from surging infections. Whether you are an NHS practice in England looking to reach the revised 85\% UDA target for this quarter, a private practice trying to balance increased demand and cancellations, or a mixed practice trying to juggle multiple factors Dentally is here to support you.

By leveraging the power of cloud-based software Dentally, you can help patients, providing them with the perfect platform to inform your practice of any cancellations due to isolation, giving them the ability to cancel, reschedule and book their own appointments all from the comfort of their home. Your patients can trust in your practices organisational processes, and you can know ahead of time of any gaps in your diary, keeping the chairs full, staff safe and the business running seamlessly.

With late cancellations leaving gaps in your diary, having practice management software in place that can support you and help you fill these gaps is essential.

The Dentally diary is easy to use and simple to understand - helping you effectively identify gaps in your practitioner's day. By using Dentally's features such as the Appointments Report and Recalls, you can fill these appointment times with people who could be overdue for vital oral care, who are not self-isolating.

It is important during this time that you prepare for more than one practitioner to be absent at once - the worst-case scenario. Times of absence can be blocked out in the Dentally calendar so practitioners having to isolate will not accidentally receive new appointments during their isolation period.

Your team can easily manage diaries, keep patients up to date and reschedule appointments as efficiently as possible. Assisted by several tools within Dentally, designed to take the stress out of daily practice life, you can be confident that your practice is prepared to deal with unexpected absences.

If your team need to work remotely because they have to self-isolate but are still able to work as they are symptomfree or because they are looking after a relative at home - with Dentally, they can! With the correct permission settings, your practice team can easily and securely access Dentally from home, meaning they can triage appointments, organise diaries and communicate with patients via outbound calls - even when they are self-isolating.

Dentally understands that when times are challenging, having practice management software that supports you whilst taking some of the stress out of daily practice life is fundamental.

That's why Dentally's cloud-based software provides the ultimate solution, helping you keep in contact with your patients and them with you so you can maintain an efficient and safe working environment for both practice staff and patients too. Speak to the team now at hello@dentally.co to find out more about how they can help you during this tricky time.

For more information visit: https:// dentally.co/ or call 02038565610.

\title{
Cosmetic waste disposal done correctly
}

More and more dentists are inching their way into the world of cosmetics, which means they'll be able to offer a more comprehensive range of treatments to patients - in a safe and sterile environment.
Introducing non-surgical cosmetic treatments, such as botulinum toxin injections, means dentists need an appropriate disposal system for any cytostatic waste (the purple waste stream on

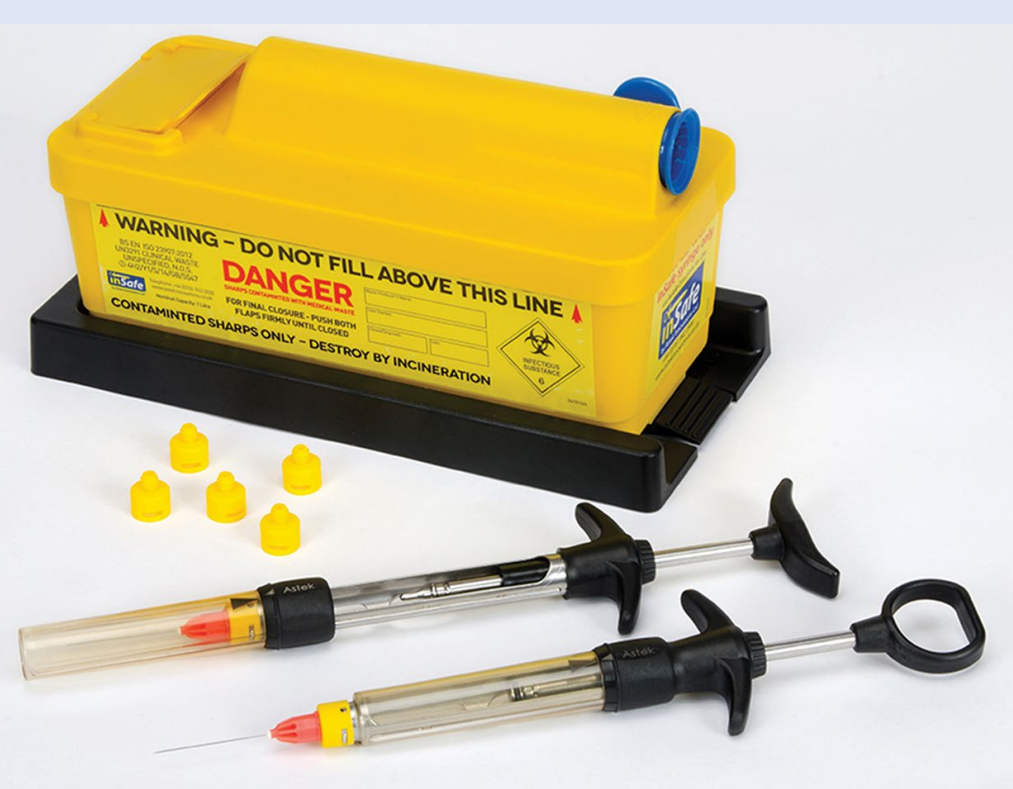

the Department of Health's colour-coded guide to best practice waste disposal). Initial Medical provides a range of waste disposal solutions, such as innovative colour-coded sharps bins.

For needles used to administer botulinum toxin injections, dentists will need the purple-coded sharps bin - they are available in sizes from 0.4 litres to 35 litres and are designed to fit seamlessly into your dental practice. They even include mounting brackets to place the sharps bin in the most optimal position. For needles used to administer anaesthetic, professionals will need one of our yellow colour-coded sharps bins.

Giving your patients more choice doesn't have to mean more confusion - with Initial Medical's colour-coded sharps bins, keeping patients and staff protected is as easy as ever.

For further information visit www.initial. co.uk/medical or Tel: 08708504045 . 